\title{
Long-term dietary-exposure to non-coplanar PCBs induces behavioral disruptions in adult zebrafish and their offspring
}

\author{
Samuel Péan ${ }^{a}$, Tarek Daouk $^{b}$, Caroline Vignet $^{b}$, Laura Lyphout $^{\mathrm{a}}$, Didier Leguay ${ }^{\mathrm{a}}$, Véronique Loizeau $^{\mathrm{C}}$, \\ Marie-Laure Bégout $^{\mathrm{a}, *}$, Xavier Cousin ${ }^{\mathrm{b}, \mathrm{d}}$
}

\footnotetext{
a Ifremer, Laboratoire Ressources Halieutiques, Place Gaby Coll, BP 7, 17137 L'Houmeau, France

${ }^{b}$ Ifremer, Laboratoire d'Ecotoxicologie, Place Gaby Coll, BP 7, 17137 L'Houmeau, France

' Ifremer, Laboratoire de Biogéochimie des Contaminants Organiques, Centre de Brest, BP 70, 29280 Plouzané, France

d INRA LPGP, Campus de Beaulieu, 35042 Rennes, France
}

\author{
*: Corresponding author: Marie-Laure Bégout, tel.: + 33546500608 ; fax: + 33546500650 ; \\ email address : marie.laure.begout@ifremer.fr
}

\begin{abstract}
:
The use of polychlorinated biphenyls (PCBs) has been banned for several decades. PCBs have a long biological half-life and high liposolubility which leads to their bioaccumulation and biomagnification through food chains over a wide range of trophic levels. Exposure can lead to changes in animal physiology and behavior and has been demonstrated in both experimental and field analyses. There are also potential risks to high trophic level predators, including humans. A maternal transfer has been demonstrated in fish as PCBs bind to lipids in eggs. In this study, behavioral traits (exploration and free swimming, with or without challenges) of contaminated zebrafish (Danio rerio) adults and their offspring (both as five-day-old larvae and as two-month-old fish reared under standard conditions) were measured using video-tracking. Long-term dietary exposure to a mixture of non-coplanar PCBs was used to mimic known environmental contamination levels and congener composition. Eight-weekold fish were exposed for eight months at $26-28{ }^{\circ} \mathrm{C}$. Those exposed to an intermediate dose (equivalent to that found in the Loire Estuary, $\Sigma_{\mathrm{CB}}=515 \mathrm{ng} \mathrm{g}^{-1}$ dry weight in food) displayed behavioral disruption in exploration capacities. Fish exposed to the highest dose (equivalent to that found in the Seine Estuary, $\Sigma_{C B}=2302 \mathrm{ng} \mathrm{g}^{-1}$ dry weight in food) displayed an increased swimming activity at the end of the night. In offspring, larval activity was increased and two-month-old fish occupied the bottom section of the tank less often. These findings call for more long-term experiments using the zebrafish model; the mechanisms underlying behavioral disruptions need to be understood due to their implications for both human health and their ecological relevance in terms of individual fitness and survival.
\end{abstract}

\section{Highlights}

Zebrafish lifecycle exposure to PCB mixture with non-coplanar congeners Behavioral disruption in adults and progeny after 8 mo. of PCB contamination Medium dose exposed fish showed over reaction in challenging tests High dose exposed fish showed increased swimming activity at the end of night. Progeny showed hyperactivity as larvae and lower home base behavior as juvenile.

Keywords: Exploration ; Swimming activity ; Organic pollutant effects ; Generation ; Video analysis 


\section{Introduction}

Polychlorinated biphenyls (PCBs) are a family of 209 synthetic molecules composed of a biphenyl nucleus with chlorine at any, or all, of the 10 available sites [49]. The ortho, meta and para positions are important in determining the chemical properties of PCB, such as high dielectric constants, non-flammability, hydrophobic quality and chemical stability. The usefulness of these properties led PCBs to be used in mixtures for a wide range of applications from the 1930s onwards [7,14]. However, after their toxicity became recognized, PCBs were progressively banned in most developed countries during the 1980s [44]. Due to their large-scale production, extensive use and environmental persistence, these compounds have accumulated in many ecosystems all over the world; in aquatic environments they are trapped in sediments. PCB contamination continues to be a problem as compounds can be transferred from the sediment to the lower trophic levels of an ecosystem through microbial and bottom-feeder uptake. In addition to this entry into the trophic chain, constant resuspension of sediment due to human activity (for example, trawling or dredging) or natural events (such as floods) induces de novo contamination. The long biological half-life and high liposolubility of PCBs can lead to their bioaccumulation and biomagnification through food chains over a wide range of trophic levels and cause a potential risk for high trophic level predators $[13,28,29,57]$. For human and fish populations, dietary intake, especially the consumption of marine organisms, is considered one of the most important sources of PCB exposure $[36,51,60,64]$. Many local and national agencies have published recommendations to limit human consumption of fish and shellfish in contaminated coastal or freshwater areas.

Data from epidemiologic and field studies have suggested that exposure to PCBs may have adverse effects on the physiology of animals, including humans. Experimental exposure studies have been performed to understand PCB toxicity and its underlying mechanisms of action. These analyses have demonstrated alteration of reproductive, hepatic, renal and endocrine functions in both animal models and humans $[20,34,70,73,94]$.

The use of model fish species, such as medaka or zebrafish, for eco- and neurotoxicology studies has developed rapidly over the last decade $[32,33,79,83]$. These models demonstrated several alterations in physiology after PCB exposure, including changes in development and reproduction [8,21,31,47,55,56,61].

Quantitative behavior analysis is now considered to be a good indicator of organism stress response. It can be used to assess a wide set of chemical stressors in pharmacology, toxicology and ecotoxicology testing and the use of fish models in this context is now widely accepted $[16,19,59,80]$.

Direct exposure to PCBs has been shown to modify certain behavioral traits in various animals and humans $[6,26,37,65,67,71,88]$. More recently, studies have demonstrated modifications in the behavior of fish as a result of acute exposure $[43,53,54,75]$. These articles report exposure to mixtures known to content dioxin-like coplanar congeners (exact composition not given) through their addition to water and food sources. In all cases, exposure were short (about 3 weeks) and dose exposure high (1 to10 $\mu \mathrm{g}^{-1} \mathrm{I}^{-1}$ for water exposure [43,75] and 15 to125 $\mu \mathrm{g}^{-1}$ effective concentration in diet [53,54]). Described behavioral disruptions include changes in locomotor activity $[53,75]$, school size or shape disruption [54] and entry to seawater by salmon smolt [75].

Epidemiological studies on children have identified a link between PCB exposure and attention deficit/hyperactivity disorder (ADHD)[63,71]. Indirect parental contamination has also been shown using mammal models $[18,26,34,65,86]$. In mammals, embryo development 
takes place within the uterus under constant feeding through the placenta; there is therefore a potential chronic exposure of fetuses to PCBs through blood exchanges. Exposure can be prolonged through milk provided through breastfeeding. Fish are mostly precocious animals, meaning that there is no parental care of the young. As such, behavior of the young is not directly influenced by the mother"s behavior. In fish, PCBs can be transmitted from the dam to eggs[10,21].

We aimed to explore if fish behavior would be modified following chronic long-term exposure to PCB contamination during juvenile and adult life. A PCB mixture was added to their diet to mimic environmental contamination. Zebrafish were fed contaminated diets from the age of eight weeks (i.e. after sex differentiation), and for more than eight months (about half of the life of a zebrafish), using a PCB mixture which was similar in dose and congener composition to those measured in European coastal mollusks flesh. Individual responses are relevant to both ecology and the assessment of toxicity mechanisms. Therefore, tests based on individual behavior can be useful indicators of pollutant effects $[9,33,46,48,74]$. We used behavioral tests related to essential components of fish behavior in the wild. Tests on control and contaminated adult zebrafish (hereafter called F0 fish), and on their offspring (hereafter called F1 fish), measured their exploration of a novel environment and free swimming activity over 24 hours. F1 fish were studied when very young and at juvenile stages. We monitored the visual motor response $[15,24]$ in five-day-old larvae in a light/dark challenge, following a method slightly modified from that of Ali et al. (2012). This method is used extensively in the behavioral profiling of zebrafish larvae exposed to diverse compounds. In F0 fish and twomonth-old juvenile offspring, we recorded free swimming activity in a 24-hour experiment. We also analyzed vertical movement which was recorded over another 24-hour period. This behavior is important because, according to Levin et al. (2007) in a shorter and slightly different challenge, choice of position in a novel tank experiment (at upper vs. lower levels) is considered an index of anxiety. In rodents, similar indices analyze their choice of position in an elevated "plus maze" (in an open vs. closed arm) or in an open field experiment (near the wall vs. center).

\section{Materials and methods}

\subsection{Rearing conditions and $P C B$ exposure}

The present behavioral experiments carried out on adults (F0) and their offspring (F1) were part of a larger trial, described in full by Daouk et al. (2011). This study was conducted under the approval of the Animal Care Committee of France under the official license granted to M.L. Bégout (17-010).

Wild type zebrafish (unreferenced strain, Danio rerio) were bought at six weeks of age from a breeder who specializes in supplying zebrafish for ecotoxicology experiments (Élevage de la Grande Rivière, Calluire, France). PCB exposure began two weeks later. The fish were maintained and exposed to a PCB mixture at the Ifremer Fish Ecophysiology Platform (L'Houmeau, France; http://wwz.ifremer.fr/pep/), under standard conditions: at a water and room temperature of $26-28{ }^{\circ} \mathrm{C}$ and under a $14: 10$ light:dark cycle. The water used in the rearing system and during tests was a mixture of water treated by reverse-osmosis and tap water; both were filtered through dust and charcoal filters to obtain a $\mathrm{pH}$ of $7.5 \pm 0.5$ and a conductivity of $300 \pm 50 \mu \mathrm{S} . \mathrm{cm}^{-1}$. Ammonia, nitrites, and nitrates were monitored daily for two months, then weekly after this period, and remained within recommended ranges [42]. Fish were raised in a flow-through system made of 20 identical $10-\mathrm{L}$ tanks arranged in a rack. A proportion of the water was changed automatically every hour, leading to a replacement rate 
of 4 L.day ${ }^{-1} \cdot \operatorname{tank}^{-1}$. Contaminated water was collected and treated with activated charcoal before being discharged into a sewer.

Commercial food (Neo supra, pellet-size $1400 \mu \mathrm{m}$ ), obtained from Le Gouessant (France), consisted of a formulated feed containing $58 \%$ crude protein and $13 \%$ crude fat. Spiked food was prepared by slowly adding a solution containing known amounts of thirteen congeners in iso-octane (AccuStandard Inc., New Haven, USA, purity above 98\%) to food pellets whilst constantly mixing. Diets were prepared in small batches to avoid deterioration. PCB mixtures were composed of the seven indicator PCB congeners (CB-28, CB-52, CB-101, CB-118, CB138 , CB-153 and CB-180), with six others added to cover a larger range of chlorination from three to eight chlorine atoms (CB-105, CB-132, CB-149, CB-156, CB-170 and CB-194). Individual PCB congener concentrations were adjusted to known environmental ratios and determined afterwards in each batch of spiked food (methods and results in Table 1, [14]). Two diets were prepared, "PCB-medium" and "PCB-high", for which the cumulated congener concentrations were $\Sigma_{C B}=515 \mathrm{ng} \cdot \mathrm{g}^{-1}$ dry weight $(\mathrm{dw})$ and $\sum_{\mathrm{CB}}=2302 \mathrm{ng} \cdot \mathrm{g}^{-1} \mathrm{dw}$, respectively [21]. These cumulated congener concentrations correspond those recorded in medium and highly contaminated sites (in the flesh of mollusks from the Loire and Seine estuaries, respectively; data from French coastal chemical contamination survey network: $\mathrm{ROCCH}$, http://envlit.ifremer.fr). The control food exposed only to solvent is hereafter referred to as "Solvent", and the unmodified food is referred to as "Plain". Non-spiked food was also analyzed for PCB levels, and concentrations were between $<0.05 \mathrm{ng} \cdot \mathrm{g}^{-1}$ dry weight (dw) and $3.0 \mathrm{ng} \cdot \mathrm{g}^{-1} \mathrm{dw}$ (mean value calculated on five replicates) depending on the congener (see Daouk et al. 2011). In addition to the congeners included to spike the contaminated food, we measured p.p'DDE (3.50 ng/g dw). The presence of non-added congeners in non-spiked food were also assessed to ensure that dioxin-like PCBs were absent: CB-31 (not detected (nd) with a limit of detection $(\mathrm{LOD})=0.3 \mathrm{pg} / \mu \mathrm{l}), \mathrm{CB}-77$ (nd, LOD=0.64 pg/ $/ \mu \mathrm{l}), \mathrm{CB}-110(0.75$

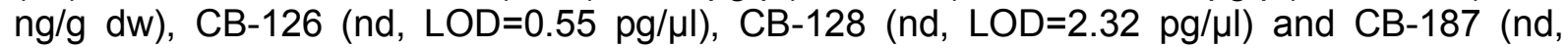
$\mathrm{LOD}=1.08 \mathrm{pg} / \mu \mathrm{l})$. Further details about the doses of each congener are available in Daouk et al. (2011). Plain food was used as a control to test for variation that could be due to the solvent or spiking procedures. Twenty 10-L tanks were used in total: three tanks for each of the Plain and Solvent control groups and seven tanks for each of the PCB-medium and PCBhigh groups. There were 30 individuals in each tank.

After 250 days of contamination, the exploration and swimming activity of adults (F0 fish, 10 mo. old) were measured and, after a further 50 days of contamination, offspring (F1 fish) were obtained by crossing males and females from the same cited treatments (for Solvent, PCB-medium and PCB-high groups). The Plain treatment group was not used for crosses as we did not see different behavioral phenotypes between Plain and Solvent (see results). Three larvae batches (for each batch, $n=30$ ) were produced from three spawns which were each obtained using three different randomly sampled pairs. These were kept in Petri dishes, in an incubator at $28^{\circ} \mathrm{C}$ and under the same photoperiod as adults. Visual motor responses to a five minute light/dark challenge were monitored at the age of five days. Other larvae batches originating from more than 10 randomly formed pairs were reared under normal conditions in the facility, using untreated food (as described above); their swimming activity was measured at two months of age (see next section).

\subsection{Behavioral experiments}

Behavioral experiments on F0 adults and two-month-old F1 were done in a dedicated room, kept at $27 \pm 1^{\circ} \mathrm{C}$ with a 14:10 light:dark photoperiod synchronized to that of the rearing room to minimize unwanted effects. „Daylight" was started at 08:30 and there were no twilight periods. 
Due to a biased sex ratio in favor of females in the F0 experimental groups, we were not able to evaluate a possible sex-effect for all tests. Females were used for the T-Maze exploration test (12 fish per treatment). For the 24-h swimming activity test, other groups of fish composed of six females and six males were used per treatment. Different fish were used in each test. Behavioral experiments on F1 groups were performed in five-day-old larvae and in two-month-old fish for which sex could not be determined due to their size. All fish (from the four treatment groups (F0 adults) or three treatments groups (two-month-old F1)) were sampled equally from all replicates, size matched as best as possible, and measured for weight $(\mathrm{mg})$ and length $(\mathrm{mm})$ after the tests.

\subsubsection{F0 - T-maze exploration}

This experiment was adapted from Ninkovic and Bally-Cuif (2006) using the same T-maze built with four arms (see supplementary material Figure S1) but running only one exploration trial per individual since we were not investigating learning ability in this experiment. For each trial, one of the long arms was blocked with a removable partition and the other was used as start area. Half of each treatment group $(n=6)$ were tested in the deep area on the left and the other half $(n=6)$ were tested in deep area on the right, avoiding any laterality bias.

The T-maze was subdivided into two areas. The deep area $(10 \mathrm{~cm}$ deep $\times 23 \mathrm{~cm}$ wide $\times 23$ $\mathrm{cm}$ long) had black walls and contained marbles, plastic grass and an opaque plastic tube that could be used for shelter. This area was considered to be a favorable zone [58]. The shallow area $(5 \mathrm{~cm}$ deep) included both the start arm which led to the deep area (46 cm long) and the other arm which led in the opposite direction to the deep area; the total length of these two arms was $66 \mathrm{~cm}$. Fish were individually placed into a 1-L tank in the experiment room on the day before the experiment. For each trial, a single fish was removed from its tank and gently placed in the start area of the longest arm of the T-maze. Five minutes of exploration activity was immediately recorded. The variables recorded were velocity, mobility, latency to reach the deep area, number of exits from the deep area and time spent in the deep area.

\subsubsection{F0: $24-\mathrm{h}$ swimming activity}

Fish from each treatment and session were randomly placed in 3-L tanks $(24.5 \times 15 \times 13.5$ cm, AquaBox® 3, AQUA SCHWARZ GmbH, Göttingen, Germany), filled with $1.5 \mathrm{~L}$ of system water, to avoid tank position and session bias. The 12 tanks (four treatments, each with three fish) were isolated from neighboring tanks by opaque walls. The set-up was placed on top of an infrared apparatus (IR floor $1 \times 1 \mathrm{~m}$, Noldus, Netherlands). During the day, the room was lit with two halogen spotlights (Philips $80 \mathrm{~W}$, illumination of $30 \mathrm{~lx}$. near the tank). During the night, the spotlights were turned off and infrared light from the floor was used to record fish movements (illumination $<1 \mathrm{~lx}$. near the tank). Fish were placed in their tanks at 18:00 the day before the experiment, for one night of acclimatization. Recording started the next day at 12:30 and lasted $24 \mathrm{~h}$. We measured the distance travelled $(\mathrm{cm})$ during four experimental sessions for a total of 12 fish per treatment. The water was changed after each session.

\subsubsection{F1 larvae: Visual motor response to a light/ dark challenge}

Zebrafish larvae were individually presented with a sudden dark challenge (light off for $5 \mathrm{~min}$ ) at five days post-fertilization (dpf); video recordings were made over three periods of 5 min each: before (Light on-1, 70 Ix.), during (Light off, <1 Ix.) and after the light was turned back 
on (Light on-2). At 17:00 the day before the challenge, single larvae were transferred to the cells of a 24-well plate (Krystal 24, opaque (white) wall and clear bottom micro-plate) where they were arranged in a mixed design and visually isolated from each other. Larvae from all treatments were studied at the same time and in the same plates to avoid any trial effect. The 24-well plates were kept overnight in an incubator. The following day, two hours before the challenge, the well plate was transferred to the video acquisition room and placed on top of a size-matched infrared floor, which allowed the larvae to be filmed under both light and dark conditions. The experiment was conducted between 14:00 and 18:00, the most stable activity period in zebrafish larvae [48]. Constant IR lighting was maintained during filming using a three-way switch. The apparatus was enclosed within a lightproof and temperaturecontrolled box. Distance travelled $(\mathrm{cm})$ was recorded for 90 larvae per treatment (Solvent, PCB-medium and PCB-high).

\subsubsection{F1 (two-month-old): $24-h$ swimming activity}

We used the same set-up as described above for F0 fish. Here, we had fish from three treatments randomly placed in the 12 tanks and carried out two filming sessions. For one session, the camera was placed above the tanks to monitor horizontal movements (this top view was the same as for F0 adults). For the other session, the camera was placed in front of the tanks to record vertical movement. For this side view; the tanks were placed on a fourlevel shelf in front of the vertically placed IR floor. For each treatment we filmed 14 fish from above (top view) and 9 fish from the side (side view); Swimming path length (top view), time spent in each third of the tank section horizontally and number of passages between sections (side view) were measured.

\subsection{Data recording and analysis}

Videos were recorded with an analog camera (either a Panasonic CCTV WV-CL920A for F0 adults or an Ikegami ICD-48E for two-month-old F1 fish) with a Fujinon lens $(2.7-13.5 \mathrm{~mm})$; these were linked to a PC with an acquisition card (Euresys Picolo Series). Videos were acquired by Ethovision X.T. 7 software (Noldus, The Netherlands) at 25 frames per second. Videos for the light/dark challenge in five dpf larvae were recorded using a camera (DMK31AU03, The Imaging Sources, Germany; positioned $45 \mathrm{~cm}$ above the well plate), Fujinon lens [1.4-12.5 mm] and IC-Capture (The Imaging Sources, Germany). Track extraction and analysis were performed with Ethovision X.T. 7.

\subsection{Measured variables and statistical analysis}

The variables chosen to evaluate behavioral performances were as follows:

- Velocity $\left(\mathrm{cm} \cdot \mathrm{s}^{-1}\right)$ : the distance moved by individual fish between two consecutive $X-Y$ coordinates (per unit time; measured from their center point);

- Time when mobile (s): the duration of movement of the fish expressed as proportion of total time (\%);

- Binary coding for reaching the deep area: 0 (failure) and 1 (success);

- Latency to reach the deep area: the time (s) between the beginning of the trial and the first entry into the deep area; the number of exits from the deep area and total time spent (s) in the deep area over the 5 min trial duration. 
- Distance moved $(\mathrm{cm})$ : the distance travelled by the center point of the subject between two consecutive $X-Y$ coordinates acquired summed over a 5-min or 30-min period for the larvae light/dark challenge and 24-h experiment, respectively.

- Time spent (s) per 30-min period by a fish in the upper, mid, and bottom sections during the 24-h swimming experiment filmed in side view.

- Number of passages per 30-min period between the upper and mid section, and between the mid and bottom sections.

Data were statistically tested using Statistica 9.0 (Statsoft, USA). For each variable, a Shapiro-Wilk test was performed to check the normality and a Bartlett"s test was used to check the homogeneity of variance. All figures show mean \pm SD except otherwise mentioned. When normality or homogeneity or variance rules were not respected, MannWhitney- $U$ or Kruskal-Wallis tests [92], followed by multiple comparison of the average rank tests corrected for the number of comparisons, were performed to compare results between the four (F0) or three (F1) treatments. For the 24-h swimming activity experiment, transition periods induced an exacerbated pattern; it was thus necessary to analyze these transitional 30-min "Light-off" and "Light-on" periods separately. Remaining day and night periods were divided into blocks of six or seven 30-min period (for day 1 (D1-1 (period 12:30 to 16:00), D12 (16:00 to 19:30) and D1-3 (19:30 to 22:30)), for night (N1-1 (23:00 to 02:30), N1-2 (02:30 to 06:00) and N1-3 (06:00 to 09:00)) and for day two (D2-1 (09:30 to 12:30))). Cumulated variables (sum of distance travelled, number of passages and time spent in the bottom section) were calculated for each block. Within each of the ten blocks, Kruskal-Wallis tests were used to compare the different treatments: the distances travelled (F0 and F1), the number of passages between sections (F1) and the time spent in the bottom section (F1). Day and night results for the distance moved during the horizontal movement analysis were compared, excluding the transitional half-hour periods, using Mann-Whitney and multiple comparisons tests (as above; F0 and F1). The variable distance travelled by larvae complied with normality rules. Data were compared using a repeated-measures ANOVA and by Newman-Keuls multiple comparisons test. Treatment was considered as the within-subject factor (Solvent, PCB-medium or PCB-high) and period (Light on-1, Light off or Light on-2) as the between-subjects factor. In the T-maze experiment, proportions of fish non-exiting deep area were compared using a chi-square test. All statistical analyses were carried out at a $95 \%$ level of significance.

\section{Results}

\subsection{Fish contamination}

The experiments assessed behavioral responses of zebrafish exposed to a PCB mixture by one of two spiked diet regimes (PCB-medium and PCB-high). These groups were compared with a control treatment group where the diet was spiked with solvent alone (Solvent,) and with a fourth group of fish which were fed an unaltered diet (Plain; comparison in F0 generation only). To ensure that F0 fish were contaminated, a random sample of fish from each tank were assayed (for practical reasons, not all fish could be tested). All congeners administered in the spiked food were detected at higher concentrations in exposed F0 fish than in control fish (Loizeau V., personal communication): Similar linear PCB accumulation kinetics were observed in both male and female fish during the first three months of exposure (Fig. 1 in Daouk et al. 2011). At the time of behavioral observations, CB-153 concentration in females was highly variable ([473-1644] $\mathrm{ng} \mathrm{g}^{-1} \mathrm{dw}$ for the PCB-medium diet group and

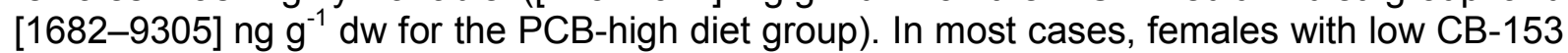
concentrations were sampled immediately after having spawned (more details in Daouk et al. 
2011). Male contamination levels were more homogeneous ([183-365] $\mathrm{ng} \mathrm{g}^{-1} \mathrm{dw}$ for the PCBmedium diet group and [1560-3141] $\mathrm{ng}^{-1} \mathrm{dw}$ for the PCB-high diet group). CB-153 concentrations in control fish (Plain and Solvent groups) were significantly lower (18.5 \pm 3.7 $\mathrm{ng}^{-1} \mathrm{dw}$ ) and there were no difference between sexes. PCBs were found to transfer to zebrafish eggs: $16.0 \pm 0.8$ and $47.6 \pm 7.8 \mathrm{ng}^{-\mathrm{g}^{-1}}$ fresh weight for PCB-medium and PCB-high diets, respectively [21].

All F0 fish used for behavioral tests showed no significant weight and length differences between treatment groups (weight: $750 \pm 75.3 \mathrm{mg}, \mathrm{KW}_{(3,96)}=1.624, \mathrm{p}=0.654$; length: $2.1 \pm$ $\left.0.3 \mathrm{~cm}, \mathrm{KW}_{(3,96)}=0.023 \mathrm{p}=0.991\right)$. Daouk et al. $(2011)$ showed there were no differences in growth between diet groups (by comparison of final weight or specific growth rate) and liver somatic- and gonad somatic indexes measured for females in each treatment showed no significant differences. Weight and length of two-month-old F1 fish were not significantly different between treatment groups (Solvent, PCB-medium and PCB-high) for either the 24-h experiment filmed in top view: (weight $320.6 \pm 55.7 \mathrm{mg}, \mathrm{KW}_{(2,54)}=2.065, \mathrm{p}=0.356$; length $\left.2.62 \pm 0.02 \mathrm{~cm}, \mathrm{KW}_{(2,54)}=2.537 \mathrm{p}=0.281\right)$ or the 24-h experiment filmed from the side view: (weight $335.7 \pm 52.7 \mathrm{mg}, \mathrm{KW}_{(2,27)}=1.019, \mathrm{p}=0.600$; length $2.64 \pm 0.09 \mathrm{~cm}, \mathrm{KW}_{(2,27)}=4.560$ $p=0.458)$. There were no instances of mortality during the behavioral experiments.

\subsection{F0:T-maze exploration}

There was data from all fish for the analysis of shallow area variables. Mean velocity was significantly different between treatments $\left(\mathrm{KW}_{(3,48)}=19.895, \mathrm{p}<0.01\right.$, Fig. 1A). Multiple comparison rank tests showed that velocity of PCB-medium group fish was significantly higher than those in Solvent, Plain and PCB-high treatment groups. The Kruskal-Wallis test with multiple comparison rank tests indicated a significant difference between treatments in terms of the proportion of time fish were mobile in the shallow area $\left(\mathrm{KW}_{(3,48)}=12.961, \mathrm{p}<\right.$ 0.01 , Fig. 1B); PCB-medium fish were significantly more mobile $(80.9 \pm 5.1 \%$ of the time $)$ than fish in other treatments ( $42 \%$ to $55 \%)$.

The second part of the analysis focused on the deep area and only concerned fish that reached this area.

Fish from PCB-medium treatment reached the deep area significantly faster (after $28.7 \pm 9.4$ s) than fish from Plain, Solvent and PCB-high treatment groups (these later groups did not differ from each other: mean time $=110 \pm 50 \mathrm{~s}, \mathrm{KW}_{(3,40)}=10.362, \mathrm{p}<0.02$, Fig. 2A). The total time spent in the deep area (not shown) or the proportion of remaining time spent their once initially reached were not significantly different between treatments $\left(\mathrm{KW}_{(3,40)}=5.96, \mathrm{p}=\right.$ 0.114; Fig. 2B). The number of exits from deep area was not different between treatments (not shown) but the proportion of fish which never exited from the deep area after having entered it differed significantly $\left(\mathrm{Chi}_{(3)}=38.36, \mathrm{p}<0.001\right)$.

\subsection{F0: $24-\mathrm{h}$ swimming activity}

By constantly monitoring of swimming activity over 24-h, we identified several periods where specific behavioral patterns corresponded to day/night and light changes. Light change in our system was abrupt and provoked an intense increase in swimming activity (distance travelled; Fig. 3A) both at lights off (22:30) and lights on (8:30). Apart from these transitional periods, distance travelled showed relatively low variation within or between day and night blocks (Fig. 3A). No sex effect was observed between distances travelled. During day blocks or during light regime changes we found no differences between treatments. Some differences did appear during the last night block (N1-3) $\left(\mathrm{KW}_{(3,48)}=15.110, \mathrm{p}=0.001\right.$; Fig $3 \mathrm{~B})$ with a higher activity in fish exposed to a PCB-high diet, and low and similar level 
measured for Plain and Solvent group fish and intermediate activity for fish exposed to the PCB-medium diet. When comparing only the photophase and the dark phase within treatment groups, no difference was observed between day and night activity level with the exception of the PCB-high treatment group which had higher activity at night $\left(\mathrm{MW}-\mathrm{U}_{(24)}=\right.$ 34.000; $p=0.028$ ). No differences were observed between Plain and Solvent controls in F0 fish; this led us to focus only on three treatments for F1 fish (Solvent, PCB-medium and PCB-high).

\subsection{F1 larvae: Visual motor response to a light/dark challenge}

Fish from all the treatments showed a similar response pattern to the light/dark challenge: between the first 5-min light period and the dark period there was a 1.4-1.9 fold increase in swimming activity (Fig. 4). When the light came on again, distance travelled decreased and was generally lower than observed during the first lit period. There was a significant treatment effect $\left(\right.$ ANOVAR $_{(6,502)}=18.670, p<0.001$; Newman-Keuls post-hoc test) during Light on-1 and Light off, when the fish in the Solvent treatment group swam less than those from contaminated groups. During Light on-2, the PCB-medium group showed the highest distance travelled; the PCB-high group reached similar levels as the F1 Solvent treatment group.

\subsection{F1: two-month-old - 24-h swimming activity - horizontal movements}

Abrupt light change provoked an intense increase in swimming activity (distance travelled; Fig. 5), both at the time of lights off and lights on (as for F0 fish). Distance travelled showed a slight decrease from D1-1 to D1-3 and a U-shape at night (Fig. 5). No treatment effects were observed during any time block. When comparing the photophase and dark phase within treatment groups, the diurnal nature of the swimming activity was confirmed for each treatment and there was no rhythmic activity disruption (Solvent $M W-U_{(28)}=32 ; p=0.002$; PCB-medium MW- $U_{(28)}=45 ; p=0.015$; PCB-high $\left.M W-U_{(28)}=0 ; p<0.001\right)$.

\subsection{F1: two-month-old - 24-h swimming activity - vertical movements}

Vertical fish position, filmed over $24 \mathrm{~h}$, showed a marked daily change; fish shifted from occupying mostly the bottom and mid sections during the photophase to having an upper section preference during the night (Fig. 6A).

The alternation of position was quantified in terms of the number of passages between sections: the highest number of section transitions was observed at the end of the night and during light on (Fig. 7). The number of passages between the upper and mid sections showed significant treatment effects during D1-1 and D1-2 and in the middle of the night (N12) (See Supplementary material, Table S1). On day 1, F1 from the PCB-high treatment group showed a significantly higher transition activity than those from the Solvent group; fish from the PCB-medium group showed an intermediate level (Fig 7A). During the night, fewer passages were recorded for the PCB-high group than the PCB-medium group although this was not different from the passages observed in the Solvent group. The number of passages between the mid and bottom sections only showed a significant treatment effect during day 1 (D1-2, Fig. 7B, Table S1). The number of passages between the upper and middle sections during the night was constantly higher than between the middle and bottom sections, and increased during the night. For the middle-bottom transitions, the PCB-high fish showed the highest number of passages during D1-2; this was higher than observed in the Solvent group but not different to that of F1from the PCB-medium treatment group. 
When focusing solely on the time spent in the bottom section (Fig. 6B), we observed significant treatment effects for the first two blocks (D1-1 and D1-2), the light on transition and day 2 period (Table S1). During the day, F1 of the PCB-high fish showed the lowest level of bottom section occupancy. D1-1, D1-2, light on and D2-1 results were significantly different from that of the Solvent fish group but not from F1 of the PCB-medium fish which were at an intermediate level. No differences were observed during the night.

\section{Discussion}

Our results demonstrate that chronic exposure of fish to diets spiked with a mixture of PCBs, devoid of coplanar dioxin-like congeners, can alter behavioral traits. Directly contaminated fish and their offspring showed changes in exploration behavior and free swimming (both in tests with and without challenge).

\subsection{Behavioral disruptions in F0 fish}

Behavioral alterations were observed in all tests of directly contaminated fish (T-maze and 24-h swimming activity experiments). These fish had been fed on diets containing PCBs: (PCB-medium: concentration of $\Sigma_{\mathrm{CB}}=515 \mathrm{ng} \cdot \mathrm{g}^{-1}$; PCB-high: concentration of $\Sigma_{C B}=2302 \mathrm{ng} \mathrm{g}^{-1}$ ) for eight months. Differential body concentrations were attained (same values and fish as in Daouk et al. 2011). CB-153 whole body concentrations in PCB-medium diet fish were below values observed for roach in the Seine river (1568 $\mathrm{ng} \cdot \mathrm{g}^{-1} \mathrm{dw}$, [17]); PCBhigh diet fish had whole body concentrations in the same range or higher than Seine river roach. Although there may be variability in the contamination levels of sub-sampled adults, we found significantly different behavioral responses between groups for the contamination range resulting from our treatments. Both observations of long term rhythmic activity and analysis of the responses to short term challenges can reveal the behavioral alterations associated with contamination.

Concerning circadian rhythm activity, we did not show a clear diurnal activity pattern which may be due to the strain and age of the F0 fish. Indeed, we have shown that older fish ( $>6$ mo) of the TU line loose marked differences between day and night locomotor activity in comparison with $A B$ line [85]. A significant increase in swimming activity was observed for PCB-high diet fish at the end of the night (during the last third). This could be interpreted as an anticipation of the forthcoming light change [4] or due to a modification of the circadian rhythm that may only occur at high PCB doses (no such alteration noted for PCB-medium diet fish). A modification of the circadian rhythm has already been reported both in vitro and in vivo after exposure to dioxin, and involves regulation of circadian genes by AhR $[30,50,52,62,76,82,89]$. Differential expression of the clock gene was detected in a brain microarray analysis of rats exposed to Aroclor 1254 [72]. Aroclor contains the dioxin-like CB126 congener which is also able to trigger AhR mediated response [35]. The diet mixture used in this experiment contains no coplanar dioxin-like compounds (the detection limit for non-ortho substituted planar dioxin-like congeners was $0.64 \mathrm{pg} / \mu \mathrm{l})[12,38]$. This suggests that other congeners are also able to trigger rhythm disruption.

In a challenging situation and with no acclimatization period (T-maze exploration experiment), behavioral disruption was observed in fish exposed to the PCB-medium diet. Overall activity was increased in the challenged fish and deviation from the response of the control groups (Plain and Solvent groups reacted in the same way) were linked to an increase in swimming activity. Fish were introduced into a novel environment which is supposedly felt to be unsafe due to the shallow depth of water and high walls forming a straight corridor. In addition to providing information on the exploratory ability of the fish, the 
swimming characteristics we recorded during exploration gave indications as to how fish coped with this novel environment. Behavior of PCB-medium fish in the shallow area of the T-maze deviated from that of all other fish, with an increase of velocity (doubled) associated with an increase in the proportion of time mobile. This shortened the time needed to reach the deep area, which was supposedly an area more favorable for the fish than the shallow corridors. In addition PCB-medium fish settled there once reached with a lower proportion of fish exiting from deep area. Thus, behavioral responses measured in the shallow area supported the idea that PCB-medium diet fish over-reacted under challenging conditions. There are few reports of hyperactivity induced by fish exposure to PCB [54,75]. Higher individual night swimming activity was found in carp exposed to Aroclor 1254 (in solution at $10 \mu \mathrm{g} \mathrm{L}^{-1}$ ); group level schooling behavior of medaka was disrupted when food was spiked with Kanechlor-400 (25 $\mu \mathrm{g} \cdot \mathrm{g}^{-1}$ and $100 \mu \mathrm{g} \cdot \mathrm{g}^{-1}$, represent 10- to 100-fold higher doses than those in the present study). These studies also used mixtures containing coplanar dioxin-like congeners (such as CB-77 and CB-126) which were absent in our study and may suggest again a different pathway of action. The convergence between our results and the literature highlights the needs for further experimentation, using low doses, to aim to identify of this possibly non-AhR pathway.

When comparing the main behavioral responses observed in fish from the PCB-medium and PCB-high diet treatments, although contamination levels of fish themselves is in agreement with PCB concentration in each diet [21], there was no reinforced increase in measured effects with increased dose. We acknowledge that the factor of contamination difference between our diets is low (4.5). The absence of a dose-response effect was also observed for the fertilization rate of these fish (lowered by $25 \%$ for both PCB-medium and PCB-high exposed fish) and in their follicle maturation rates [21]. Systematic, dose-response screening using this family of molecules is further required since a large-scale analysis of zebrafish has demonstrated dose-response or non-monotonic behavioral responses depending on chemicals [1], and non-monotonic responses have specifically been reported with PCBs $[3,11,84,87,90]$. We found that behavioral disruptions observed in fish fed the PCB-medium diet were related to response to stimulus or environmental changes whilst those of the fish fed with PCB-high diet were rhythm disruptions. The underlying mechanisms of induced behavioral change may therefore be different.

Additional studies are currently being performed to explore the roles of mono-ortho substituted congeners, and of other congeners. Research is also planned to explore the possible molecules mechanisms and modes of action that could bring about such behavioral changes.

\subsection{Behavioral disruptions in F1 fish}

In descendants exposed only via maternal transfer, both at a very early age (larvae at five days post-fertilization), and persisting into the juvenile stage, we found some significant different behavioral responses within the contamination range attained. To our knowledge, this is the first time that effects of PCBs exposure have been monitored in the offspring of contaminated fish.

At five days, most larvae organs are developed [39,68], and they already shows a complex behavioral repertoire $[2,16,66]$. Assays performed at this stage conform to ethical requirements (compliance to the $3 R$ rule, as tests at this stage and in this species are considered to be in vitro; [81]). Although light information processing involves complex mechanisms [27], the visual motor response test used to assess behavior of larvae is considered robust and better than other methods for revealing defective brain function, aberrant nervous system development and locomotor defects caused by toxic compounds $[1,40,66]$. Higher activity levels observed in larvae might be the consequence of several non- 
exclusive events. For example, developmental defects could be caused by i) exposure of embryos and larvae to PCBs after maternal transfer to the egg, ii) alteration of oocyte quality in response to maternal reaction after exposure to PCBs or iii) epigenetic imprinting of gametes which can modify their development or physiology. Of these notions, there is evidence for our first suggestion as we have shown that dietary PCBs are transferred to zebrafish eggs [21].

Here, after acclimatization to the environment for two hours without disturbance, locomotor activity levels stabilized and the first period of recording (5 min in duration, Light on-1) reflected basal activity. The larval activity level of PCB-medium progeny was slightly higher than that of PCB-high progeny; this pattern of response to concentration is similar to that described in the F0 adults. These differences remained during the change from light to dark conditions. Activity was stimulated in PCB-medium F1 fish but suppressed in PCB-high F1 fish during the Light on-2 phase. This higher challenge response from the PCB-medium larvae was also observed in the F0 adult parental fish during the T-maze challenge.

We acclimatized fish overnight before recording 24-h activity test to measure free swimming activity in the horizontal and vertical planes. In terms of horizontal distances travelled (recorded in top-view experiments), we found high diurnal swimming activity; a similar pattern was described by del Pozo et al. (2011) using another method [22]. We observed strong responses to the light transition period in fish from all treatment groups.

Regarding the vertical positioning of fish, Solvent F1 fish stayed close to the bottom during most of the photophase but preferred a near-surface position overnight. The contaminated F1 treatment group use the bottom section significantly less during the day (similar to a dosedependent monotonic suppression) and moved between sections more often in the afternoon (12:00-19:00). This was particularly evident for the PCB-high F1 fish. In the wild, zebrafish occupy the entire water column, apparently without vertical distribution bias $[25,77,78]$. In tanks however, zebrafish preferentially occupy both top and bottom layers of the tank during sleeping periods and have a marked preference for the top [91]. By recording vertical positioning continuously over $24 \mathrm{~h}$, our study provides detailed documentation of this daily spontaneous shift in vertical positioning.

The "novel diving tank test" may be used to explore stress and anxiety in juvenile or adult zebrafish, but only captures five or six consecutive minutes of activity during daytime $[23,45,69]$. A significant correlation between increased use of the lower tank section and an anxious or stressed state has been recorded in such studies. This state may be induced by different known anxiogenic drugs (such as caffeine) or relieved by anxiolytic ones (such as fluoxetine or nicotine) and was confirmed by cortisol assays $[23,45]$. Our experimental protocol undoubtedly differs from such tests but, despite this, the positioning of contaminated fish suggests a decrease of stress and/or anxiety.

Nighttime behavioral patterns have been described by several authors working on sleep or rest/wake profiles in zebrafish; the sleep state is characterized by reversible periods of immobility, increased arousal threshold and place preference [91,93]. Zebrafish respond to hypnotic drugs and are therefore a useful model for understanding the impact of sleep and sleep deprivation on cognitive functions and performance in higher vertebrates. F1 fish issued from contaminated parents showed only minor changes in their vertical positioning during the night which suggests that, contrary to the F0 fish, nocturnal behavior were not markedly affected. Further studies should be conducted to explore the cognitive functions and performance of these fish. 


\subsection{Hyperactivity induced in PCB-exposed zebrafish offspring}

Strikingly, in both F0 and F1 generations, the behavior differences reported in contaminated fish reflected an increase in some form of activity; this was visible in the challenge situation for some fish and in the day-long activity recordings for others. PCB exposure in F0 fish started at the juvenile stage and lasted over eight months. Behavior disruption may therefore reflect physiological disturbances such as endocrine disruption and metabolic imbalance. Several mechanisms such as developmental alterations or genetic imprinting, could explain both early and late behavioral disruptions in F1 fish. The activity increases found here are of particular interest as they are relevant to the established link between PCB exposure and a human behavioral deficit known as attention deficit/hyperactivity disorder (ADHD) [26,63]. The link between PCB exposure and ADHD in children was shown by epidemiological studies on children [71] and has also been noted in many mammal models [18,26,34,65,86]. ADHD may persist into human adulthood [65] and a similar persistence during aging was suggested in our fish model. It is possible that zebrafish, exposed to an environmental PCB mixture through diet, could also serve as a model to improve the understanding of the mechanisms underlying the appearance of hyperactivity in different generations. Further analyses should be conducted to evaluate the potential mechanistic relationship between PCBs and the concentration of neurotransmitters (such as dopamine and serotonin) $[5,11,41]$.

\subsection{Ecological relevance}

This work has ecological relevance as the PCB mixtures used are representative of those found to occur in European coastal areas, both in terms of congener mixtures and their concentrations [21]. The behavioral deviations reported here revealed similar trends or effects as reported in other studies of fish $[53,54,75]$. However, unlike these other studies, we did not use coplanar dioxin-like congeners (CB-77 and CB-126) in the diet mixtures. This suggests that similar behavioral disruption may be promoted through different pathways. This is important from an ecological point of view as it would imply that exposure to different environmental mixtures (related to different geographical areas) may result in similar behavioral changes. We report the first analysis of both fish contaminated during juvenile and adult life, and their offspring. Further investigations are required where dietary exposure occurs from the very first meal onwards.

\section{Conclusions}

Long-term exposure to a diet spiked with a PCB mixture devoid of non-coplanar dioxin-like congeners (akin to that found in mildly contaminated environments) induced behavioral disruption in both zebrafish parents and their offspring. Directly contaminated parents, at the higher tested diet dose, showed an increase in nocturnal activity. However, both levels of contamination may have ecological consequences due to energy budget imbalances or increased predation risks. Changes in nocturnal activity may also be related to a general desynchronization that, in turn, could impair reproductive behavior and also cause an impact at the population level. Larvae of contaminated parents showed increased activity which was partly maintained though to adulthood. In two-month-old fish, the behavior difference was associated with a decrease in bottom-section occupancy of the tank and an increase in the number of section transitions. This may be due to weakened homebase behavior and/or a loss of behavioral inhibition, also leading to increased predation risk. 
This study was supported by Ifremer and Région Poitou-Charentes (CPER and PhD fellowships for Samuel Péan and Caroline Vignet), the Conseil Général de la Charente Maritime (PhD fellowship for Tarek Daouk) and EC2CO-Cytrix (grant GénérationPOP to Xavier Cousin). We also thank two anonymous referees for their helpful remarks.

\section{References}

[1] S. Ali, D.L. Champagne, M.K. Richardson, Behavioral profiling of zebrafish embryos exposed to a panel of 60 water-soluble compounds, Behav Brain Res 228 (2012) 272-283.

[2] S. Ali, D.L. Champagne, H.P. Spaink, M.K. Richardson, Zebrafish embryos and larvae: a new generation of disease models and drug screens, Birth Defects Res C Embryo Today 93 (2011) 115-33.

[3] N. Aluru, E.H. Jorgensen, A.G. Maule, M.M. Vijayan, PCB disruption of the hypothalamuspituitary-interrenal axis involves brain glucocorticoid receptor downregulation in anadromous Arctic charr, Am J Physiol Regul Integr Comp Physiol 287 (2004) R787-93.

[4] A. Aranda, J.A. Madrid, F.J. Sanchez-Vazquez, Influence of light on feeding anticipatory activity in goldfish, J Biol Rhythms 16 (2001) 50-7.

[5] V. Berg, J.L. Lyche, C. Karlsson, B. Stavik, R. Nourizadeh-Lillabadi, N. Hårdnes, J.U. Skaare, P. Alestrøm, E. Lie, E. Ropstad, Accumulation and effects of natural mixtures of persistent organic pollutants (POP) in zebrafish after two generations of exposure, Journal of Toxicology and Environmental Health, Part A: Current Issues 74 (2011) 407-423.

[6] D.F. Berger, J.P. Lombardo, P.M. Jeffers, A.E. Hunt, B. Bush, A. Casey, F. Quimby, Hyperactivity and impulsiveness in rats fed diets supplemented with either Aroclor 1248 or PCB-contaminated St. Lawrence river fish, Behavioural Brain Research 126 (2001) 1-11.

[7] A. Beyer, M. Biziuk, Environmental fate and global distribution of polychlorinated biphenyls, Rev Environ Contam Toxicol 201 (2009) 137-58.

[8] K. Billsson, L. Westerlund, M. Tysklind, P.-E. Olsson, Developmental disturbances caused by polychlorinated biphenyls in zebrafish (Brachydanio rerio), Marine Environmental Research 46 (1998) 461-464.

[9] S.R. Blechinger, R.C. Kusch, K. Haugo, C. Matz, D.P. Chivers, P.H. Krone, Brief embryonic cadmium exposure induces a stress response and cell death in the developing olfactory system followed by long-term olfactory deficits in juvenile zebrafish, Toxicol Appl Pharmacol 224 (2007) 72-80.

[10] X. Bodiguel, V. Loizeau, A.-M. Le Guellec, F. Roupsard, X. Philippon, C. Mellon-Duval, Influence of sex, maturity and reproduction on PCB and p,p'DDE concentrations and repartitions in the European hake (Merluccius merluccius, L.) from the Gulf of Lions (N.W. Mediterranean), The Science of the Total Environment 408 (2009) 304-311.

[11] J. Boix, O. Cauli, H. Leslie, V. Felipo, Differential long-term effects of developmental exposure to polychlorinated biphenyls 52,138 or 180 on motor activity and neurotransmission. Gender dependence and mechanisms involved, Neurochem Int 58 (2011) 69-77.

[12] J.P. Boon, J.C. Duinker, Kinetics of polychlorinated biphenyl (PCB) components in juvenile sole (Solea solea) in relation to concentrations in water and to lipid metabolism under conditions of starvation, Aquatic Toxicology 7 (1985) 119-134.

[13] K. Borgå, G.W. Gabrielsen, J.U. Skaare, Biomagnification of organochlorines along a Barents Sea food chain, Environmental Pollution (Barking, Essex: 1987) 113 (2001) 187-198.

[14] K. Breivik, A. Sweetman, J.M. Pacyna, K.C. Jones, Towards a global historical emission inventory for selected PCB congeners--a mass balance approach. 1. Global production and consumption, Sci Total Environ 290 (2002) 181-98.

[15] H.A. Burgess, M. Granato, Modulation of locomotor activity in larval zebrafish during light adaptation, Journal of Experimental Biology 210 (2007) 2526-2539.

[16] D.L. Champagne, C.C.M. Hoefnagels, E. de Kloet, K. Richardson, Translating rodent behavioral repertoire to zebrafish (Danio rerio): Relevance for stress research, Behavioural Brain Research 214 (2010) 332-342.

[17] M. Chevreuil, A.-M. Carru, A. Chesterikoff, P. Boët, E. Tales, J. Allardi, Contamination of fish from different areas of the river Seine (France) by organic (PCB and pesticides) and metallic 
( $\mathrm{Cd}, \mathrm{Cr}, \mathrm{Cu}, \mathrm{Fe}, \mathrm{Mn}, \mathrm{Pb}$ and $\mathrm{Zn}$ ) micropollutants, Science of The Total Environment 162 (1995) 31-42.

[18] A. Colciago, L. Casati, O. Mornati, A.V. Vergoni, A. Santagostino, F. Celotti, P. Negri-Cesi, Chronic treatment with polychlorinated biphenyls (PCB) during pregnancy and lactation in the rat Part 2: Effects on reproductive parameters, on sex behavior, on memory retention and on hypothalamic expression of aromatase and 5alpha-reductases in the offspring, Toxicol Appl Pharmacol 239 (2009) 46-54.

[19] R. Creton, Automated analysis of behavior in zebrafish larvae, Behavioural Brain Research 203 (2009) 127-136.

[20] K.M. Crofton, P.R.S. Kodavanti, E.C. Derr-Yellin, A.C. Casey, L.S. Kehn, PCBs, Thyroid hormones, and ototoxicity in rats: Cross-fostering experiments demonstrate the impact of postnatal lactation exposure, Toxicological Sciences 57 (2000) 131-140.

[21] T. Daouk, T. Larcher, F. Roupsard, L. Lyphout, C. Rigaud, M. Ledevin, V. Loizeau, X. Cousin, Long-term food-exposure of zebrafish to PCB mixtures mimicking some environmental situations induces ovary pathology and impairs reproduction ability, Aquat Toxicol 105 (2011) 270-8.

[22] A. del Pozo, J.A. Sanchez-Ferez, F.J. Sanchez-Vazquez, Circadian rhythms of self-feeding and locomotor activity in zebrafish (Danio rerio), Chronobiol Int 28 (2011) 39-47.

[23] R.J. Egan, C.L. Bergner, P.C. Hart, J.M. Cachat, P.R. Canavello, M.F. Elegante, S.I. Elkhayat, B.K. Bartels, A.K. Tien, D.H. Tien and others, Understanding behavioral and physiological phenotypes of stress and anxiety in zebrafish, Behav Brain Res 205 (2009) 38-44.

[24] F. Emran, J. Rihel, J.E. Dowling, A behavioral assay to measure responsiveness of zebrafish to changes in light intensities, Journal of visualized experiments : JoVE (2008).

[25] R.E. Engeszer, L.A. Da Barbiano, M.J. Ryan, D.M. Parichy, Timing and plasticity of shoaling behaviour in the zebrafish, Danio rerio, Animal Behaviour 74 (2007) 1269-1275.

[26] P.A. Eubig, A. Aguiar, S.L. Schantz, Lead and PCBs as Risk Factors for Attention Deficit Hyperactivity Disorder, Environ Health Perspect (2010).

[27] A.M. Fernandes, K. Fero, A.B. Arrenberg, S.A. Bergeron, W. Driever, H.A. Burgess, Deep brain photoreceptors control light-seeking behavior in zebrafish larvae, Curr Biol 22 (2012) 2042-7.

[28] A.T. Fisk, K.A. Hobson, R.J. Norstrom, Influence of chemical and biological factors on trophic transfer of persistent organic pollutants in the northwater polynya marine food web, Environmental Science \& Technology 35 (2001) 732-738.

[29] A.T. Fisk, R.J. Norstrom, C.D. Cymbalisty, D.C.G. Muir, Dietary accumulation and depuration of hydrophobic organochlorines: Bioaccumulation parameters and their relationship with the octanol/water partition coefficient, Environmental Toxicology and Chemistry 17 (1998) 951961.

[30] R.W. Garrett, T.A. Gasiewicz, The aryl hydrocarbon receptor agonist 2,3,7,8tetrachlorodibenzo-p-dioxin alters the circadian rhythms, quiescence, and expression of clock genes in murine hematopoietic stem and progenitor cells, Mol Pharmacol 69 (2006) 2076-83.

[31] A.C. Grimes, K.N. Erwin, H.A. Stadt, G.L. Hunter, H.A. Gefroh, H.-J. Tsai, M.L. Kirby, PCB126 exposure disrupts zebrafish ventricular and branchial but not early neural crest development, Toxicological Sciences 106 (2008) 193-205.

[32] A.J. Hill, H. Teraoka, W. Heideman, R.E. Peterson, Zebrafish as a model vertebrate for investigating chemical toxicity, Toxicol Sci 86 (2005) 6-19.

[33] D.E. Hinton, S.W. Kullman, R.C. Hardman, D.C. Volz, P.-J. Chen, M. Carney, D.C. Bencic, Resolving mechanisms of toxicity while pursuing ecotoxicological relevance?, Marine Pollution Bulletin 51 (2005) 635-648.

[34] E. Holene, I. Nafstad, J.U. Skaare, T. Sagvolden, Behavioural hyperactivity in rats following postnatal exposure to sub-toxic doses of polychlorinated biphenyl congeners 153 and 126, Behavioural Brain Research 94 (1998) 213-224.

[35] B.A. Jensen, C.M. Reddy, R.K. Nelson, M.E. Hahn, Developing tools for risk assessment in protected species: Relative potencies inferred from competitive binding of halogenated aromatic hydrocarbons to aryl hydrocarbon receptors from beluga (Delphinapterus leucas) and mouse, Aquat Toxicol 100 (2010) 238-45.

[36] H.R. Johansen, J. Alexander, O.J. Rossland, S. Planting, M. Lovik, P.I. Gaarder, W. Gdynia, K.S. Bjerve, G. Becher, PCDDs, PCDFs, and PCBs in human blood in relation to consumption of crabs from a contaminated Fjord area in Norway, Environ Health Perspect 104 (1996) 75664. 
[37] M. Kakeyama, C. Tohyama, Developmental neurotoxicity of dioxin and its related compounds, Ind Health 41 (2003) 215-30.

[38] N. Kannan, S. Tanabe, T. Wakimoto, R. Tatsukawa, Coplanar polychlorinated biphenyls in aroclor and kanechlor mixtures, J Assoc Off Anal Chem 70 (1987) 451-4.

[39] C.B. Kimmel, W.W. Ballard, S.R. Kimmel, B. Ullmann, T.F. Schilling, Stages of embryonic development of the zebrafish, Developmental Dynamics 203 (1995) 253-310.

[40] D. Kokel, J. Bryan, C. Laggner, R. White, C.Y. Cheung, R. Mateus, D. Healey, S. Kim, A.A. Werdich, S.J. Haggarty and others, Rapid behavior-based identification of neuroactive small molecules in the zebrafish, Nat Chem Biol 6 (2010) 231-237.

[41] J.A. Kreiling, R. Creton, C. Reinisch, Early embryonic exposure to polychlorinated biphenyls disrupts heat-shock protein 70 cognate expression in zebrafish, J Toxicol Environ Health A 70 (2007) 1005-13.

[42] C. Lawrence, The husbandry of zebrafish (Danio rerio): A review, Aquaculture 269 (2007) 120.

[43] D.T. Lerner, B.T. Björnsson, S.D. McCormick, Effects of aqueous exposure to polychlorinated biphenyls (Aroclor 1254) on physiology and behavior of smolt development of Atlantic salmon, Aquatic Toxicology 81 (2007) 329-336.

[44] G. Letz, The Toxicology of PCB's - An Overview for Clinicians, West J Med 138 (1983) 534540.

[45] E.D. Levin, Z. Bencan, D.T. Cerutti, Anxiolytic effects of nicotine in zebrafish, Physiol Behav 90 (2007) 54-8.

[46] E. Linney, L. Upchurch, S. Donerly, Zebrafish as a neurotoxicological model, Neurotoxicology and Teratology 26 (2004) 709-718.

[47] J.L. Lyche, R. Nourizadeh-Lillabadi, C. Almaas, B. Stavik, V. Berg, J.U. Skare, P. Alestrom, E. Ropstad, Natural mixtures of persistent organic pollutants (POP) increase weight gain, advance puberty, and induce changes in gene expression associated with steroid hormones and obesity in female zebrafish, J Toxicol Environ Health A 73 (2010) 1032-57.

[48] R.C. MacPhail, J. Brooks, D.L. Hunter, B. Padnos, T.D. Irons, S. Padilla, Locomotion in larval zebrafish: Influence of time of day, lighting and ethanol, NeuroToxicology 30 (2009) 52-58.

[49] V.A. McFarland, J.U. Clarke, Environmental occurrence, abundance, and potential toxicity of polychlorinated biphenyl congeners: considerations for a congener-specific analysis, Environmental Health Perspectives 81 (1989) 225-239.

[50] B.E. McIntosh, J.B. Hogenesch, C.A. Bradfield, Mammalian Per-Arnt-Sim proteins in environmental adaptation, Annu Rev Physiol 72 (2010) 625-45.

[51] D. Muir, T. Savinova, V. Savinov, L. Alexeeva, V. Potelov, V. Svetochev, Bioaccumulation of PCBs and chlorinated pesticides in seals, fishes and invertebrates from the White Sea, Russia, The Science of The Total Environment 306 (2003) 111-131.

[52] M. Mukai, T.M. Lin, R.E. Peterson, P.S. Cooke, S.A. Tischkau, Behavioral rhythmicity of mice lacking AhR and attenuation of light-induced phase shift by 2,3,7,8-tetrachlorodibenzo-pdioxin, J Biol Rhythms 23 (2008) 200-10.

[53] K. Nakayama, Y. Oshima, K. Hiramatsu, T. Honjo, Alteration of general behavior of male medaka, Oryzias latipes, exposed to tributyltin and/or polychlorinated biphenyls, Journal of Faculty of Agriculture, Kyushu University 49 (2004) 85-92.

[54] K. Nakayama, Y. Oshima, K. Hiramatsu, Y. Shimasaki, T. Honjo, Effects of Polychlorinated Biphenyls on the schooling behavior of japanese medaka (Oryzias latipes), Environmental Toxicology and Chemistry 24 (2005) 2588-2593.

[55] K. Nakayama, Y. Oshima, K. Nagafuchi, T. Hano, Y. Shimasaki, T. Honjo, Early-life-stage toxicity in offspring from exposed parent medaka, Oryzias latipes, to mixtures of tributyltin and polychlorinated biphenyls, Environ Toxicol Chem 24 (2005) 591-6.

[56] K. Nakayama, N. Sei, I.C. Handoh, Y. Shimasaki, T. Honjo, Y. Oshima, Effects of polychlorinated biphenyls on liver function and sexual characteristics in Japanese medaka (Oryzias latipes), Mar Pollut Bull 63 (2011) 366-9.

[57] E. Nfon, I.T. Cousins, Interpreting time trends and biomagnification of PCBs in the Baltic region using the equilibrium lipid partitioning approach, Environmental Pollution (Barking, Essex: 1987) 144 (2006) 994-1000.

[58] J. Ninkovic, L. Bally-Cuif, The zebrafish as a model system for assessing the reinforcing properties of drugs of abuse, Methods 39 (2006) 262-274.

[59] W. Norton, L. Bally-Cuif, Adult zebrafish as a model organism for behavioural genetics, BMC Neuroscience 11 (2010) 90. 
[60] M. Nyman, J. Koistinen, M.L. Fant, T. Vartiainen, E. Helle, Current levels of DDT, PCB and trace elements in the Baltic ringed seals (Phoca hispida baltica) and grey seals (Halichoerus grypus), Environ Pollut 119 (2002) 399-412.

[61] S. Örn, P.L. Andersson, L. Förlin, M. Tysklind, L. Norrgren, The impact on reproduction of an orally administered mixture of selected PCBs in zebrafish (Danio rerio), Archives of Environmental Contamination and Toxicology 35 (1998) 52-57.

[62] M. Pesonen, M. Korkalainen, J.T. Laitinen, T.B. Andersson, O. Vakkuri, 2,3,7,8Tetrachlorodibenzo-p-dioxin alters melatonin metabolism in fish hepatocytes, Chem Biol Interact 126 (2000) 227-40.

[63] K. Polanska, J. Jurewicz, W. Hanke, Review of current evidence on the impact of pesticides, polychlorinated biphenyls and selected metals on attention deficit / hyperactivity disorder in children, Int J Occup Med Environ Health 26 (2013) 16-38.

[64] G. Pompa, F. Caloni, M.L. Fracchiolla, Dioxin and PCB contamination of fish and shellfish: assessment of human exposure. Review of the international situation, Vet Res Commun 27 Suppl 1 (2003) 159-67.

[65] D.C. Rice, Parallels between attention deficit hyperactivity disorder and behavioral deficits produced by neurotoxic exposure in monkeys, Environ Health Perspect 108 Suppl 3 (2000) 405-8.

[66] J. Rihel, D.A. Prober, A. Arvanites, K. Lam, S. Zimmerman, S. Jang, S.J. Haggarty, D. Kokel, L.L. Rubin, R.T. Peterson and others, Zebrafish behavioral profiling links drugs to biological targets and rest/wake regulation, Science 327 (2010) 348-51.

[67] C.S. Roegge, S.L. Schantz, Motor function following developmental exposure to PCBS and/or MEHG, Neurotoxicol Teratol 28 (2006) 260-77.

[68] A.L. Rubinstein, Zebrafish: from disease modeling to drug discovery, Curr Opin Drug Discov Devel 6 (2003) 218-23.

[69] J. Sackerman, J.J. Donegan, C.S. Cunningham, N.N. Nguyen, K. Lawless, A. Long, R.H. Benno, G.G. Gould, Zebrafish behavior in novel environments: effects of acute exposure to anxiolytic compounds and choice of Danio rerio line, Int J Comp Psychol 23 (2010) 43-61.

[70] S. Safe, Clinical correlates of environmental endocrine disruptors, Trends in Endocrinology and Metabolism 16 (2005) 139-144.

[71] S.K. Sagiv, S.W. Thurston, D.C. Bellinger, P.E. Tolbert, L.M. Altshul, S.A. Korrick, Prenatal organochlorine exposure and behaviors associated with attention deficit hyperactivity disorder in school-aged children, Am J Epidemiol 171 (2010) 593-601.

[72] N.A. Sazonova, T. Dasbanerjee, F.A. Middleton, S. Gowtham, S. Schuckers, S.V. Faraone, Transcriptome-wide gene expression in a rat model of attention deficit hyperactivity disorder symptoms: Rats developmentally exposed to polychlorinated biphenyls, Am J Med Genet B Neuropsychiatr Genet 156 (2011) 898-912.

[73] L.M. Schell, M.V. Gallo, Relationships of putative endocrine disruptors to human sexual maturation and thyroid activity in youth, Physiol Behav 99 (2010) 246-53.

[74] E. Scherer, Behavioural responses as indicators of environmental alterations: approaches, results, developments, Journal of Applied Ichthyology 8 (1992) 122-131.

[75] K. Schmidt, G.B.O. Staaks, S. Pflugmacher, C.E.W. Steinberg, Impact of PCB mixture (Aroclor 1254) and TBT and a mixture of both on swimming behavior, body growth and enzymatic biotransformation activities (GST) of young carp (Cyprinus carpio), Aquatic Toxicology 71 (2005) 49-59.

[76] S. Shimba, Y. Watabe, Crosstalk between the AHR signaling pathway and circadian rhythm, Biochem Pharmacol 77 (2009) 560-5.

[77] R. Spence, M.K. Fatema, M. Reichard, K.A. Huq, M.A. Wahab, F. Ahmed, C. Smith, The distribution and habitat preferences of the zebrafish in Bangladesh, Journal of Fish Biology 69 (2006) 1435-1448.

[78] R. Spence, G. Gerlach, C. Lawrence, C. Smith, The behaviour and ecology of the zebrafish, Danio rerio, Biological Reviews 83 (2008) 13-34.

[79] J.M. Spitsbergen, M.L. Kent, The state of the art of the zebrafish model for toxicology and toxicologic pathology research--advantages and current limitations, Toxicologic Pathology 31 (2003) 62-87.

[80] P.J. Steenbergen, M.K. Richardson, D.L. Champagne, The use of the zebrafish model in stress research, Prog Neuropsychopharmacol Biol Psychiatry 35 (2011) 1432-51.

[81] U. Strahle, S. Scholz, R. Geisler, P. Greiner, H. Hollert, S. Rastegar, A. Schumacher, I. Selderslaghs, C. Weiss, H. Witters and others, Zebrafish embryos as an alternative to animal 
experiments-A commentary on the definition of the onset of protected life stages in animal welfare regulations, Reprod Toxicol (2011).

[82] S.A. Tischkau, C.D. Jaeger, S.L. Krager, Circadian clock disruption in the mouse ovary in response to 2,3,7,8-tetrachlorodibenzo-p-dioxin, Toxicol Lett 201 (2011) 116-22.

[83] C. Ton, Y. Lin, C. Willett, Zebrafish as a model for developmental neurotoxicity testing, Birth Defects Research Part A: Clinical and Molecular Teratology 76 (2006) 553-567.

[84] B. Ulbrich, R. Stahlmann, Developmental toxicity of polychlorinated biphenyls (PCBs): a systematic review of experimental data, Archives of Toxicology 78 (2004) 252-268.

[85] C. Vignet, M.-L. Bégout, S. Péan, L. Lyphout, D. Leguay, X. Cousin, Systematic Screening of Behavioral Responses in Two Zebrafish Strains, Zebrafish (2013) in press.

[86] A. Vitalone, A. Catalani, C. Cinque, V. Fattori, P. Matteucci, A.R. Zuena, L.G. Costa, Longterm effects of developmental exposure to low doses of PCB 126 and methylmercury, Toxicology Letters 197 (2010) 38-45.

[87] G.A. Wayman, D. Yang, D.D. Bose, A. Lesiak, V. Ledoux, D. Bruun, I.N. Pessah, P.J. Lein, PCB-95 promotes dendritic growth via ryanodine receptor-dependent mechanisms, Environ Health Perspect 120 (2012) 997-1002.

[88] G. Winneke, Developmental aspects of environmental neurotoxicology: lessons from lead and polychlorinated biphenyls, J Neurol Sci 308 (2011) 9-15.

[89] C.X. Xu, S.L. Krager, D.F. Liao, S.A. Tischkau, Disruption of CLOCK-BMAL1 transcriptional activity is responsible for aryl hydrocarbon receptor-mediated regulation of Period1 gene, Toxicol Sci 115 (2010) 98-108.

[90] D. Yang, K.H. Kim, A. Phimister, A.D. Bachstetter, T.R. Ward, R.W. Stackman, R.F. Mervis, A.B. Wisniewski, S.L. Klein, P.R. Kodavanti and others, Developmental exposure to polychlorinated biphenyls interferes with experience-dependent dendritic plasticity and ryanodine receptor expression in weanling rats, Environ Health Perspect 117 (2009) 426-35.

[91] T. Yokogawa, W. Marin, J. Faraco, G. Pezeron, L. Appelbaum, J. Zhang, F. Rosa, P. Mourrain, E. Mignot, Characterization of sleep in zebrafish and insomnia in hypocretin receptor mutants, PLoS Biol 5 (2007) e277.

[92] J.H. Zar, Biostatistical analysis, Editoin Edition, Prentice-Hall, Englewood Cliffs, NJ, 1984.

[93] I.V. Zhdanova, Sleep in zebrafish, Zebrafish 3 (2006) 215-26.

[94] K.E. Zimmer, A.C. Gutleb, J.L. Lyche, E. Dahl, I.C. Oskam, A. Krogenaes, J.U. Skaare, E. Ropstad, Altered stress-induced cortisol levels in goats exposed to polychlorinated biphenyls (PCB 126 and PCB 153) during fetal and postnatal development, J Toxicol Environ Health A 72 (2009) 164-72. 


\section{Figures}

Figure 1: F0 adult swimming activity measured in the shallow area during the T-maze test. $A$ ) Velocity measured in the shallow area. B) Proportion of time mobile (mean \pm SD; different letters indicate significant differences among treatments; $n=12$ per treatment).

\section{Figure 1}

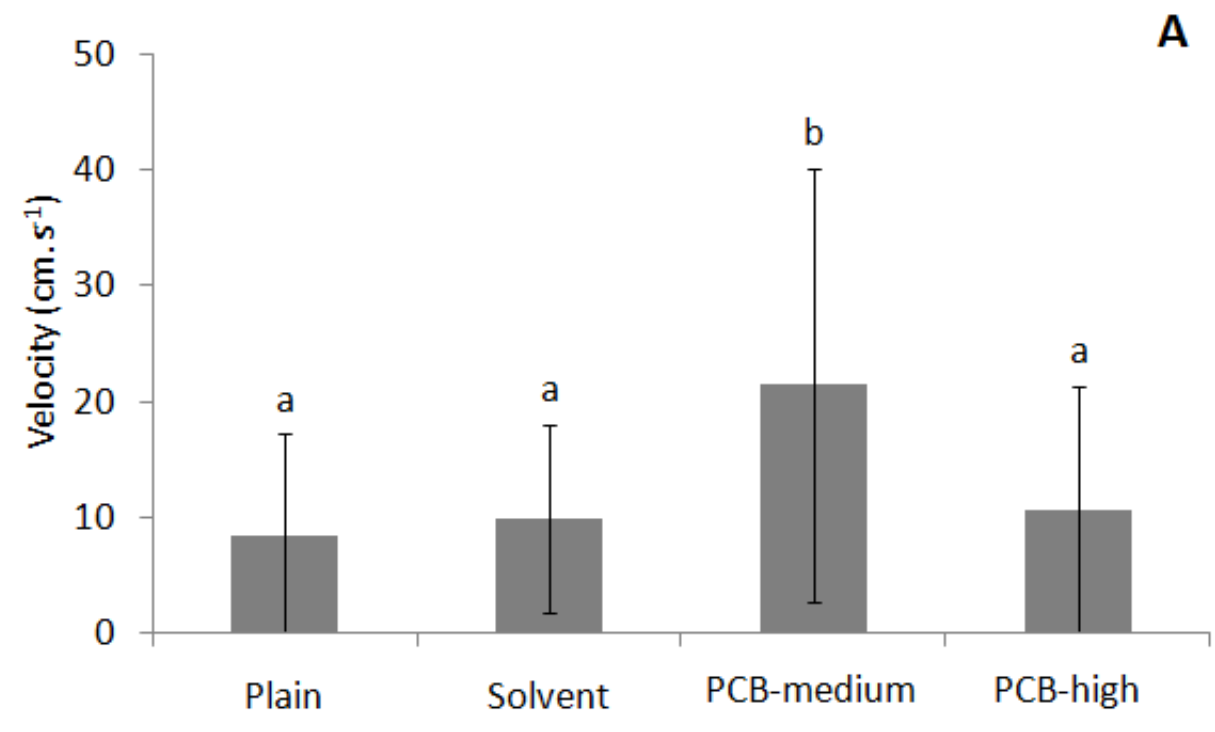

B

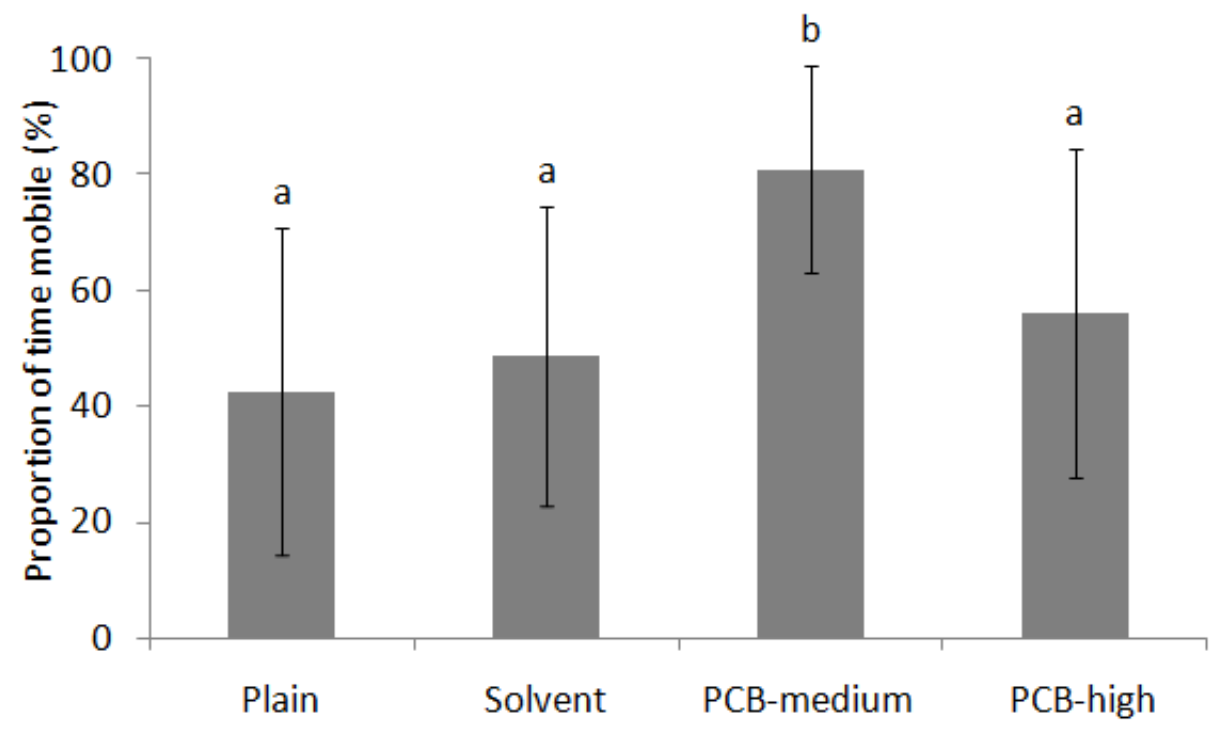


Figure 2: F0 adult swimming activity measured in the deep area during the T-maze test. A) Latency to reach the deep area. B) Proportion of time spent in the deep area once reached (dark grey bars) and of fish non-exiting the deep area (light grey bars). Sample size depends on the number of fish that reached the deep area (Plain: $n=8$, Solvent: $n=9$, PCB-medium: $n=12$ and PCB-high: $n=$ 11).

\section{Figure 2}
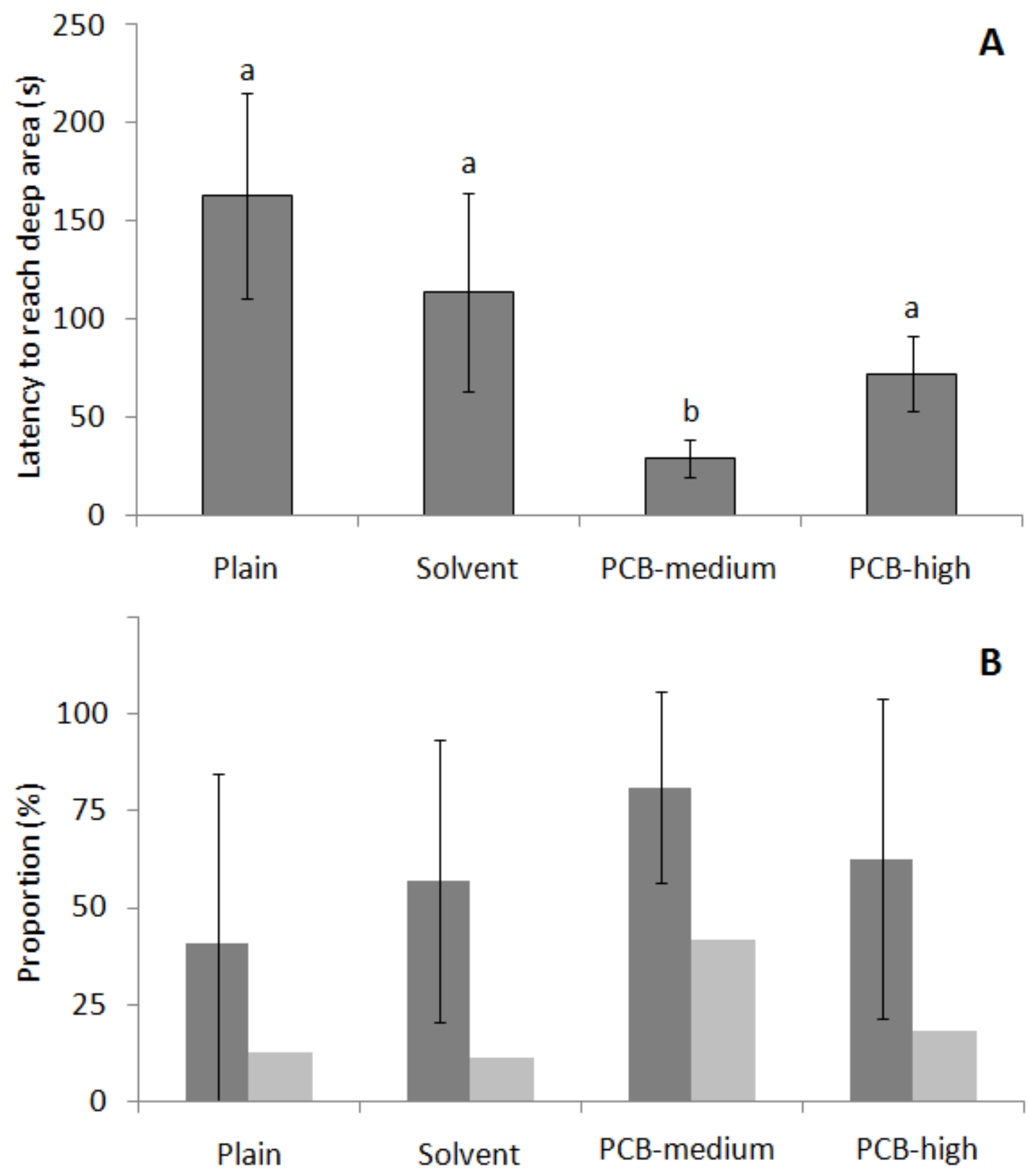
Figure 3: Distance travelled by F0 adults during the 24-h swimming activity test. A) Mean distance travelled per 30-min period. B) Average distance travelled $(\mathrm{cm} / 30 \mathrm{~min})$ for each block of the day and each treatment. Periods were divided into blocks of six or seven 30-min periods for day 1 (D11 to D1-3) night (N1-1 to N1-3) and day 2 (D2-1). The greyscale rule indicates photoperiod; L. off: Light off; L. on: Light on. Data are mean \pm SD; $n=12$ per treatment; different letters indicate significant differences among treatments and within block.

\section{Figure 3}
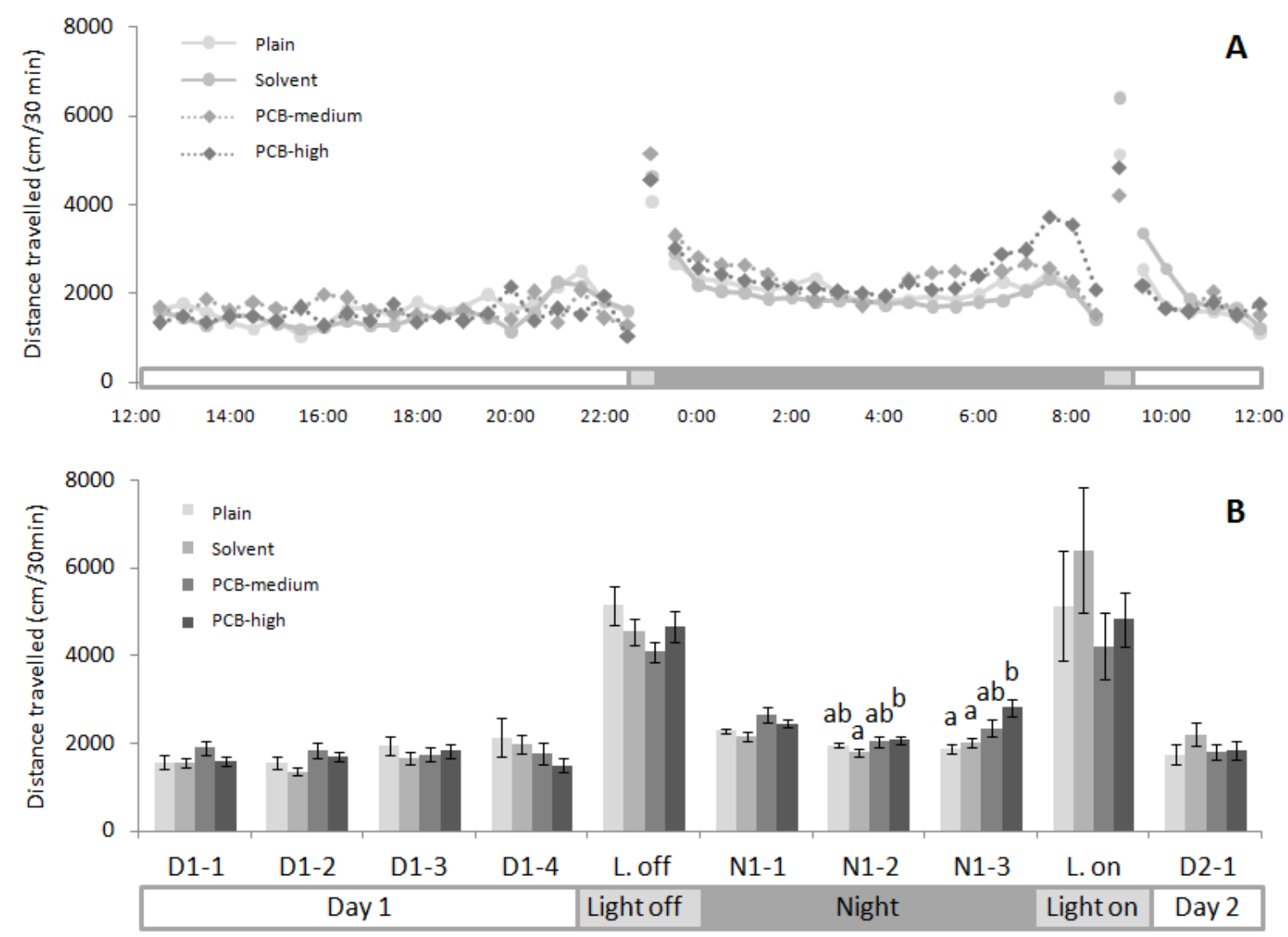
Figure 4: Swimming activity is increased in F1 larvae from contaminated parents. Average distance travelled by five-day-old larvae in 5-min bouts with light on or off (mean \pm SEM; different letters indicate significant differences among treatments; $n=90$ per treatment).

\section{Figure 4}

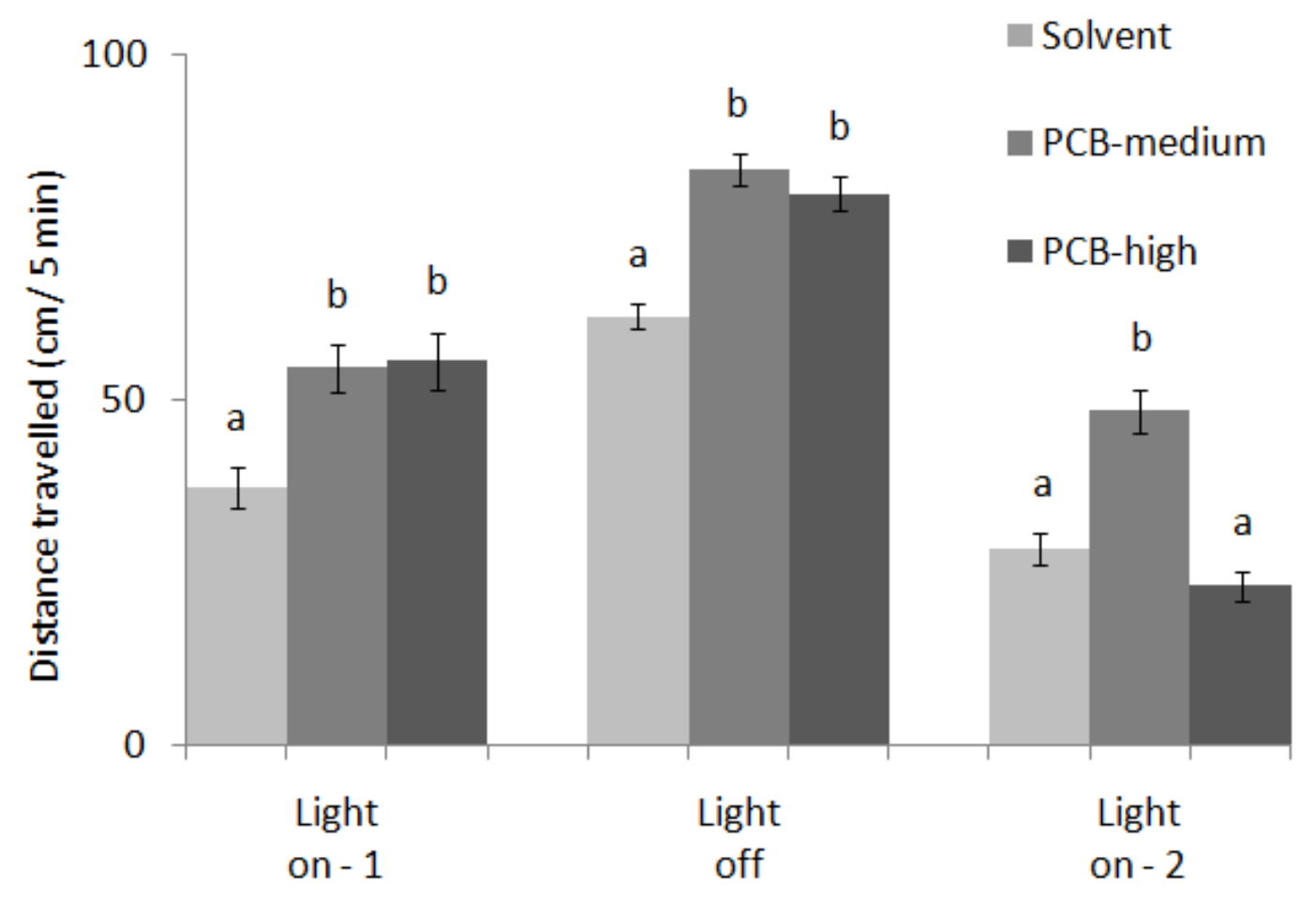


Figure 5: Distance travelled by two-month-old F1 fish during the 24-h swimming activity test. Average distance travelled $(\mathrm{cm} / 30 \mathrm{~min})$ for each block of the day and each treatment. The greyscale rule below the graph indicates photoperiod and blocks of the day (as in Fig. 3) (mean \pm SD; $n=14$ per treatment; different letters indicate significant differences among treatments and within block; $\mathrm{L}$. off $=$ Light off and L. on = Light on).

Figure 5

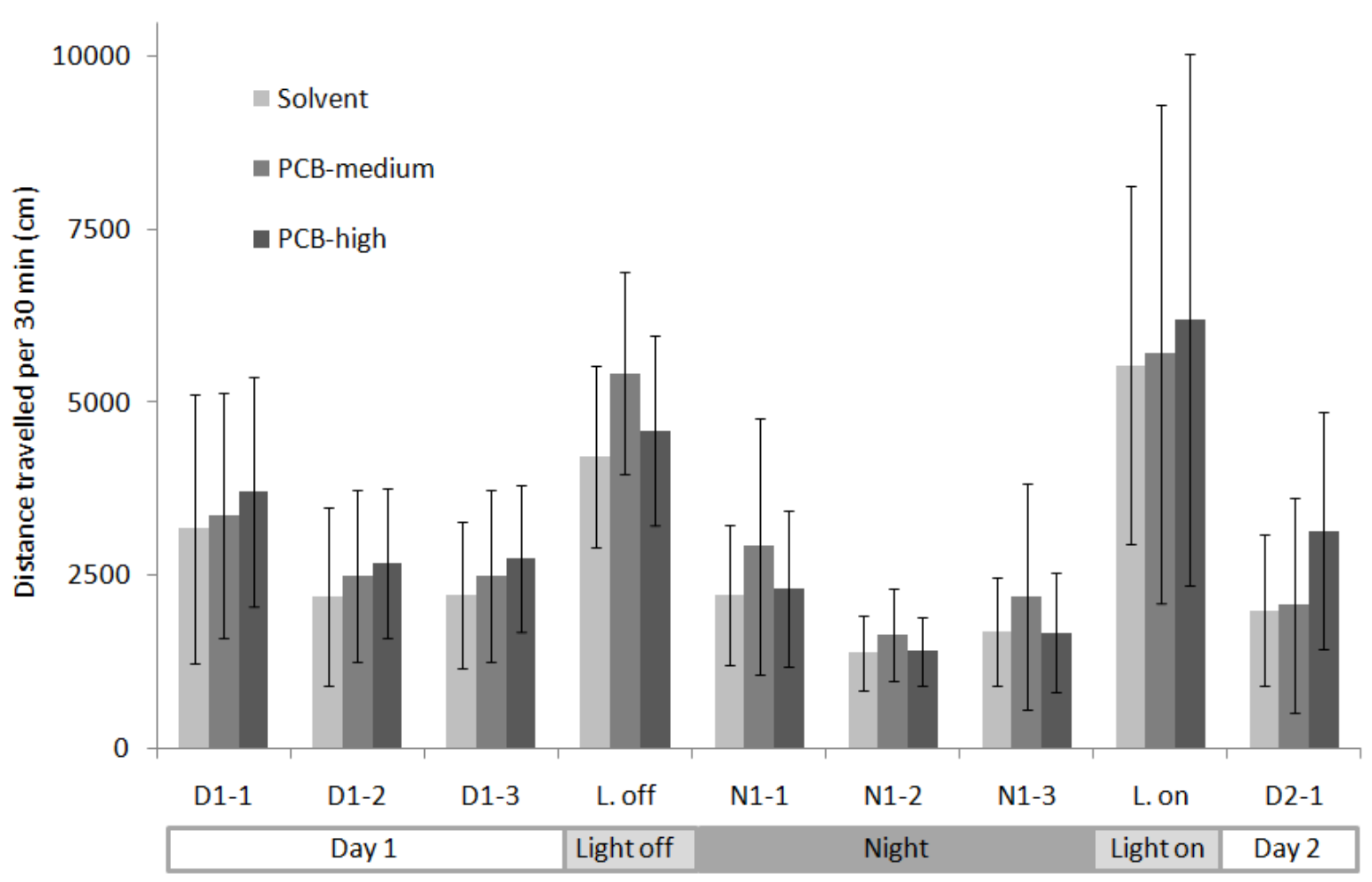


Figure 6: A. Vertical space occupancy by two-month-old F1 fish during the 24-h swimming activity test. A) Relative proportion of time (\%) spent in the bottom, mid and upper sections of a 3-L tank by two-month-old F1. B) Time spent in the bottom section, averaged for each block and each treatment. The greyscale rule below the graph indicates photoperiod and blocks of the day (mean \pm $\mathrm{SD} ; \mathrm{n}=9$ per treatment; different letters indicates significant differences among treatments and within block; L. off = Light off and L. on = Light on).

\section{Figure 6A}

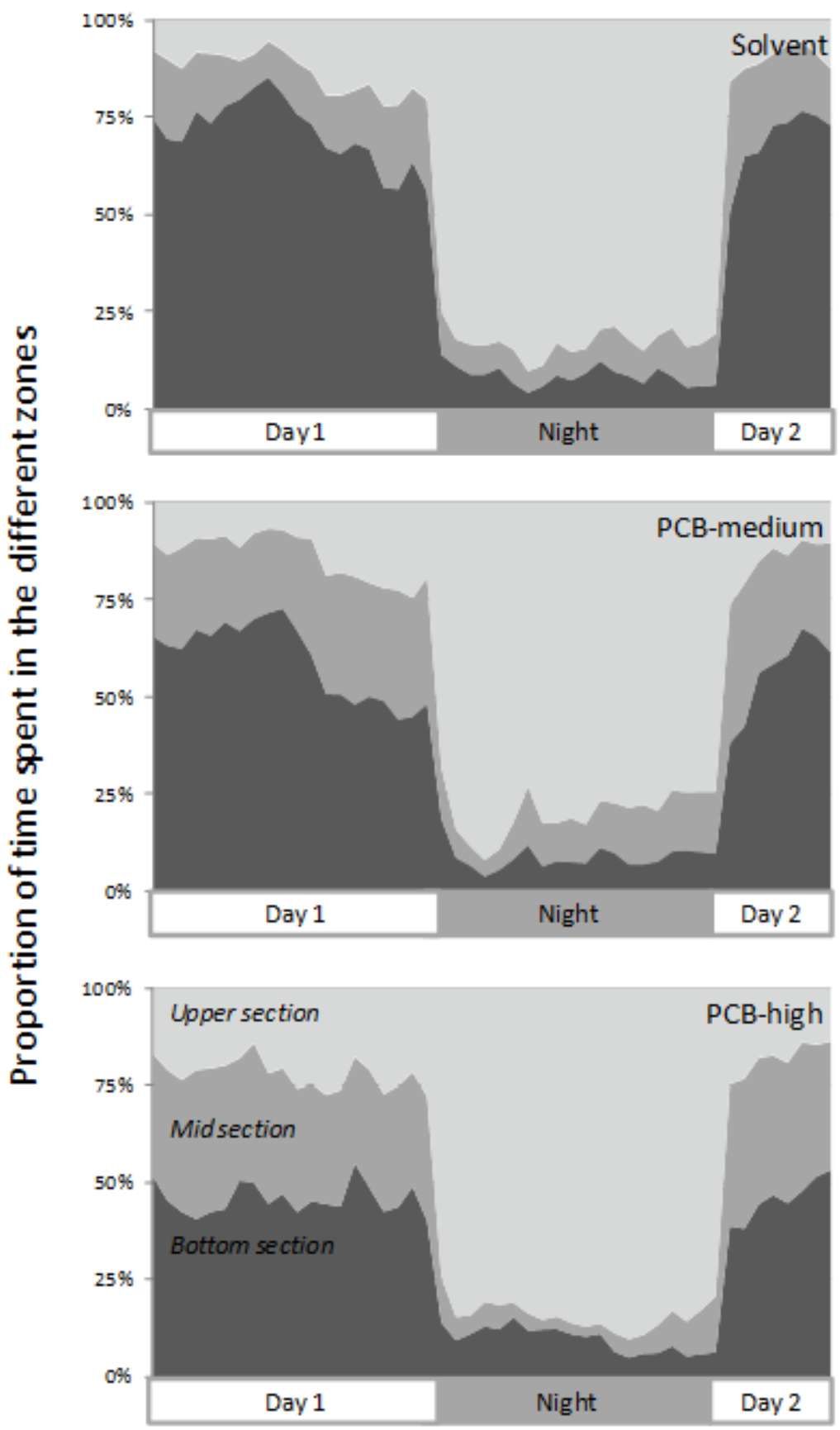


Figure 6B

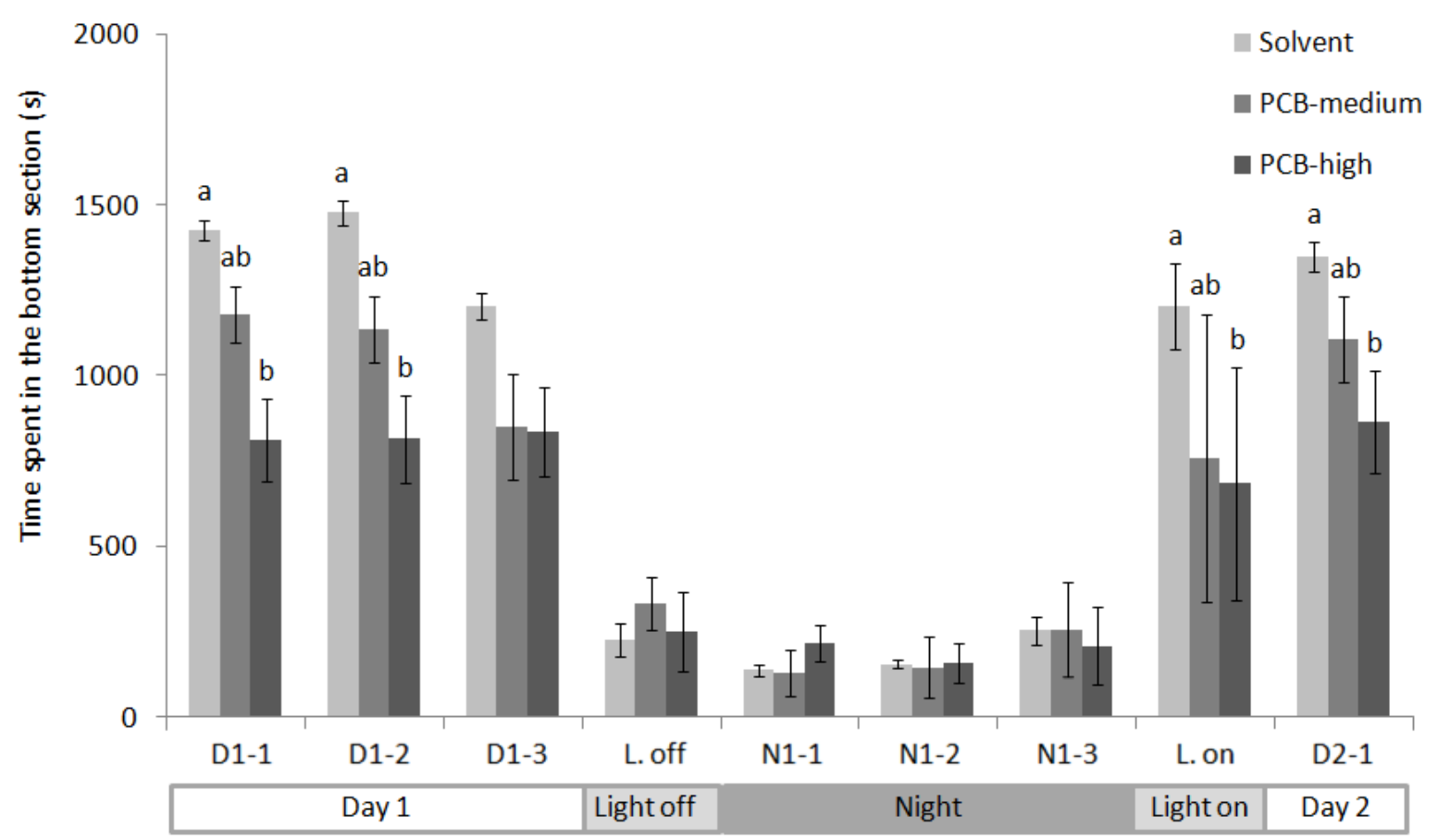


Figure 7: Number of passages between sections by two-month-old F1 fish during the 24-h swimming activity test. A) Average number of passages between upper and mid sections of the tank. B) Average number of passages between mid and bottom sections of the tank for each block of the day and each treatment. The greyscale rule below the graph indicates photoperiod and blocks of the day (mean $\pm \mathrm{SD} ; \mathrm{n}=9$ per treatment; different letters indicate significant differences among treatments and within block; L. off $=$ Light off and L. on = Light on).

\section{Figure 7}
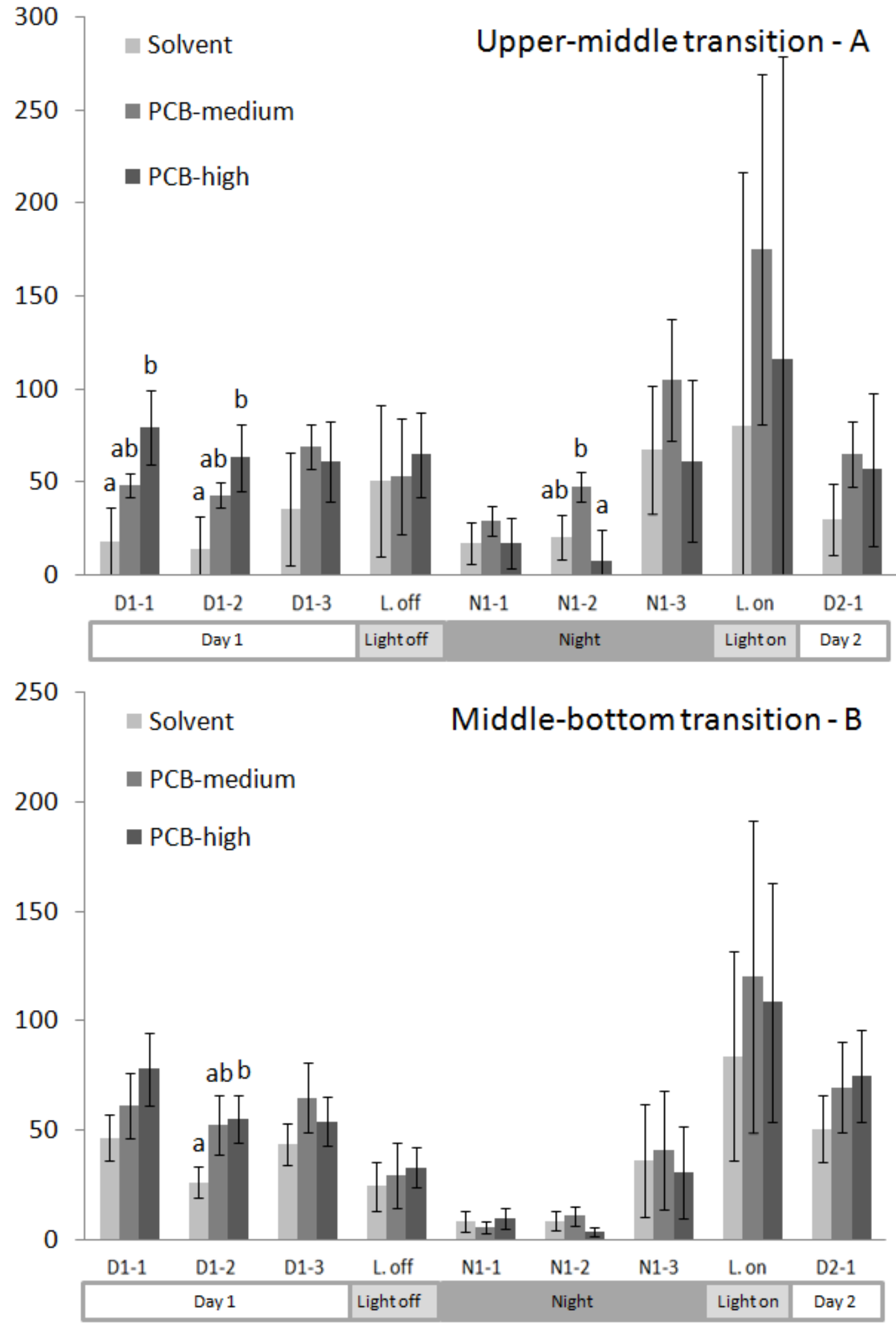